\title{
Influence of the Hydrolysis and Condensation Time on the Preparation of Hybrid Materials
}

\author{
Evelisy Cristina de Oliveira Nassor, Lilian Rodrigues Ávila, Paula Fabiana dos Santos Pereira, \\ Katia Jorge Ciuffi, Paulo Sérgio Calefi, Eduardo José Nassar* \\ University of Franca, Av. Dr. Armando Salles Oliveira, 201, CP 82, \\ CEP 14404-600, Franca, SP, Brazil
}

Received: January 9, 2010; Revised: January 12, 2011

\begin{abstract}
This work describes the hydrolytic sol-gel synthesis of silica particles prepared by a modified Stöber route, using the organofunctionalized alkoxide 3-methacryloxypropyl trimethoxysilane and tetraethylorthosilicate. The silica particles were obtained through basic catalysis from a mixture of precursor alkoxides, water, and isopropyl alcohol. Samples were prepared with change to the pre-hydrolysis and condensation time of tetraethylortosilicate, followed by addition of the organosilane 3-methacryloxypropyl trimethoxysilane. Eu ${ }^{3+}$ ions were added to the alkoxides mixture, in order to obtain structural information about the silica particles. The samples were centrifuged, washed with ethanol, and dried at $50{ }^{\circ} \mathrm{C}$. The xerogel was analyzed by thermogravimetric analysis, photoluminescence, transmission electron microscopy, and infrared spectroscopy. The photoluminescence spectra revealed $\mathrm{Eu}^{3+}$ lines characteristic of the ion ${ }^{5} \mathrm{D}_{0} \rightarrow{ }^{7} \mathrm{~F}_{\mathrm{J}(\mathrm{J}=0,1,2,3,4)}$ transition. The thermogravimetric curve and infrared spectra confirmed the presence of the alkoxide organic group and water in the materials. Transmission electron microscopy showed spherical particles with varied size. The time of pre-hydrolysis and condensation as well as the addition of 3-methacryloxypropyl trimethoxysilane affect the final shape and size of the materials, so they can be used as control factors.
\end{abstract}

Keywords: sol-gel, hybrids, spherical silica particles, europium III, 3-methacrylxypropylmethoxysilane

\section{Introduction}

Silica nanoparticles occupy a prominent position in scientific research because of their easy preparation and wide uses in industrial applications, such as pigments, pharmaceuticals, thermal insulators, humidity sensors ${ }^{1}$, catalysis ${ }^{2}$, thin films ${ }^{3}$, biomaterials ${ }^{4}$, and composite materials ${ }^{5}$. The quality of some of these products is highly dependent on the size and size distribution of these particles ${ }^{1}$.

In 1968, Stöber et al. $^{6}$ reported a pioneering method for the synthesis of spherical and monodispersed silica nanoparticles from aqueous alcohol solutions of silicon alkoxides in the presence of ammonia as catalyst; different sizes of silica nanoparticles ranging from $50 \mathrm{~nm}$ to $1 \mu \mathrm{m}$ were prepared, with a narrow particle size distribution $^{1}$. In a recent study, we prepared silica particles with $2 \mu \mathrm{m}$ using a modification of Stöber's methodology ${ }^{7}$. The particle size depends on the type of silicon alkoxide; alcohol, ammonia and water concentrations; and reaction temperature ${ }^{1}$. This method opens up the possibility for the production of organically modified particles by introducing compounds of the $\mathrm{R}_{\mathrm{n}} \mathrm{Si}\left(\mathrm{OR}^{\prime}\right)_{4-\mathrm{n}}(n=1-3$; $\mathrm{R}$ and $\mathrm{R}^{\prime}=$ alkyls) type into the starting mixture. In fact, $\mathrm{Si}-\mathrm{C}$ displays a high hydrolytic and thermal stability. Modified silicas are of interest in many fields of chemistry ${ }^{8}$. Organic compounds and metal ions, such as rare-earth ions ${ }^{7-10}$, can be selectively absorbed. This is a valuable asset in the immobilization of metal complexes for use as catalyst centers. In the biochemical field, enzymes can be immobilized, and nanostructured composites with enhanced interfacial properties can be produced ${ }^{8}$.

Several investigators have focused on the study of silica particle formation and growth mechanisms ${ }^{11}$. Two models, namely monomer addition and controlled aggregation, have been proposed to elucidate the chemical and physical growth mechanisms of silica. These models divide the formation of silica into two events: nucleation and growth. The two models use different approaches to describe particle growth. The model involving monomer addition supports a LaMer-like mechanism whereby, after an initial burst of nucleation, growth occurs through the addition of hydrolyzed monomers to the particle surface. The second model, controlled aggregation, is based on continuous nucleation throughout the reaction. These primary particles or nuclei cluster together or merge with larger aggregates to eventually produce a narrow size distribution ${ }^{12}$.

Bogush and Zukoski ${ }^{13}$ employed a primary nucleus aggregation model to describe particle formation and growth phenomena. In this case, after the silica supersaturation generated by the hydrolysis and condensation of tetraethyl orthosilicate (TEOS) exceeds a certain level, a huge number of primary nuclei of silica are suddenly nucleated and quickly aggregate to form stable particles ${ }^{12-15}$.

Thereafter, the stable particles grow on the basis of aggregation of primary nuclei. If the primary nuclei nucleated in the supersaturation are consumed mostly for the growth of stable particles, the further formation of stable particles (secondary stable particles) is prevented. This would seem to explain why monodispersed spherical silica particles are obtained ${ }^{13}$.

In the mathematical model of Matsoukas and Gulari ${ }^{13}$, particle formation and growth are similarly depicted. Here, if a sufficient number of stable particles are generated in the initial stage, no further stable particle formation should occur, resulting in monodispersed particles. Otherwise, secondary stable particles would be formed due to renewed highly built-up supersaturation ${ }^{12,13,15}$. 
The nature of the hydrolysis and condensation of alkoxides has been investigated for a long time. The several conditions can affect the size, morphology, molecular weight, and other characteristics of the silica particles. The modified organosilane as well as the hydrolysis and condensation time can change the silica properties, too.

In this work, we prepared silica particles by modifying the prehydrolysis and condensation time of the tetraethylorthosilicate (TEOS) and addition time of the 3-methacryloxypropyl trimethoxysilane (MPTMS). The resulting xerogel structure was characterized by thermal analysis (TG/DTA/DSC), photoluminescence (PL), transmission electron microscopy (TEM), and infrared spectroscopy (FT-IR).

\section{Experimental}

\subsection{Preparation of silica nanoparticles}

Silica particles were prepared by the sol-gel route using the organofunctionalized alkoxides MPTMS and TEOS in a 1:1 molar ratio, and also using the alkoxide TEOS only. The silica was obtained from a mixture of water and isopropyl alcohol in a 1:1 molar ratio; saturated ethanol ammonium solution was used as catalyst. The reaction was accomplished under magnetic stirring for 1 hour, at a temperature around $20^{\circ} \mathrm{C}$. To this end, $0.710 \mathrm{~mL}$ TEOS and $0.756 \mathrm{~mL}$ MPTMS were added to the solution. $1 \% \mathrm{Eu}^{3+}$ ions, in ethanol solution $0.1 \mathrm{~mol} . \mathrm{L}^{-1}$, were added to the silica, to obtain structural information about the silica matrix. The $\mathrm{EuCl}_{3}$ was prepared from europium III oxide $\left(0.8798 \mathrm{~g} \mathrm{Eu}_{2} \mathrm{O}_{3}\right.$ dissolved in $\left.\mathrm{HCl} 6 \mathrm{~mol}^{-\mathrm{L}^{-1}}\right)$ and calcined at $900{ }^{\circ} \mathrm{C}$ for 2 hours. The concentration of the final ethanolic solution was $0.1 \mathrm{~mol} . \mathrm{L}^{-1}$.

Six samples were produced with different TEOS pre-hydrolysis and condensation times. The first sample was prepared by adding MPTMS soon after the addition of TEOS. Four of the five remaining samples were prepared by waiting for $5,15,30$, and 45 minutes before MPTMS addition, respectively. The sixth sample was prepared without MPTMS. All the samples were centrifuged, washed with ethanol, and dried at $50{ }^{\circ} \mathrm{C}$ for 1 day.

\subsection{Characterization}

Thermal Analysis (TG/DTA/DSC) was carried out on a SDT Q600 Simultaneous DTA-TG thermal analyzer (TA Instruments) in nitrogen atmosphere, at a heating rate of $20^{\circ} \mathrm{C} / \mathrm{min}$, from 25 to $1000{ }^{\circ} \mathrm{C}$.

The photoluminescence (PL) data were obtained with a Spex Fluorolog II spectrofluorometer at room temperature. The emission was recorded at $22.5^{\circ}$ (front face) from the excitation beam.

The morphology of the system was investigated using transmission electron microscopy (TEM) (Philips CM 200 TEM, operating voltage $200 \mathrm{kV}$ ) to examine a drop of power suspension deposited on a copper grid. The crystallinity was investigated by electron diffraction in the same equipment, and energy-dispersive $\mathrm{X}$-ray spectroscopy (EDS) was used to identify the elements present in the samples.

The infrared (FTIR) spectra were obtained with a Nicolet Protege 460 spectrophotometer in $\mathrm{KBr}$ pellets.

\section{Results and Discussion}

The characterizations revealed the effect of the TEOS alkoxide hydrolysis and condensation time and the addition of organosilane MPTMS on sample structure.

Figure 1 shows TEM images of the samples prepared by adding MPTMS and $\mathrm{Eu}^{3+}$ ions $0,5,15,30$, and 45 minutes after the addition of TEOS, as well as the image of the sample obtained without the addition of MPTMS.

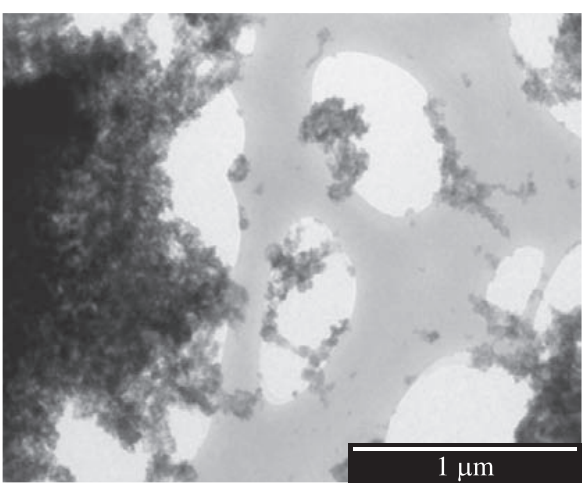

(a)

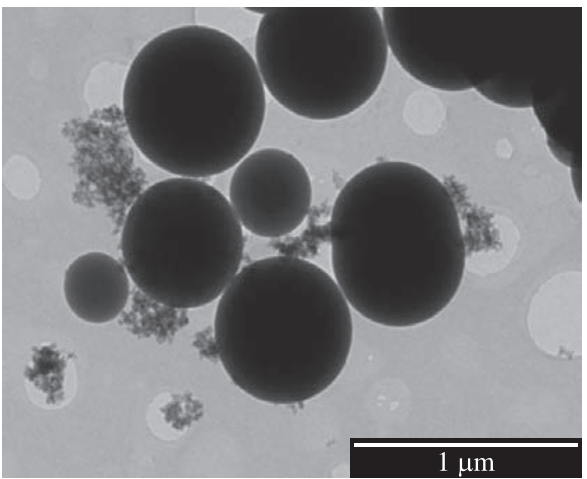

(d)

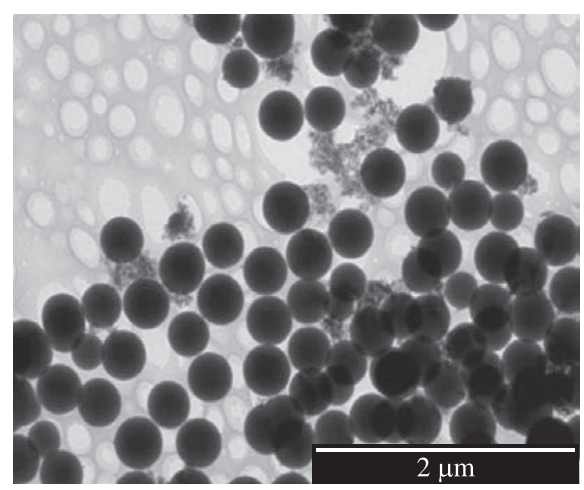

(b)

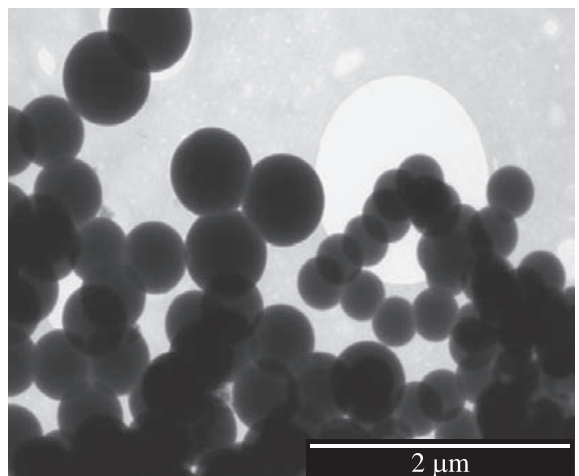

(e)

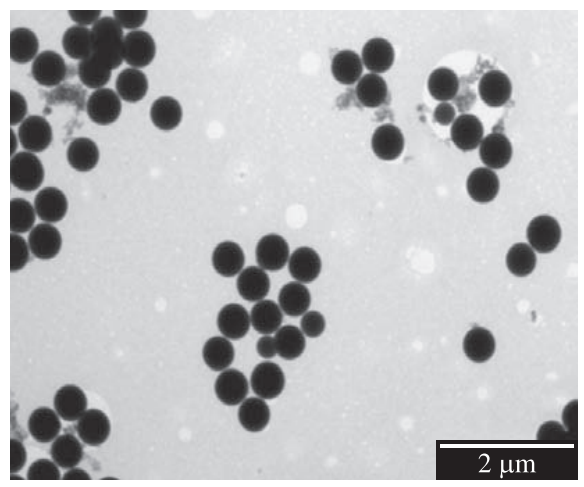

(c)

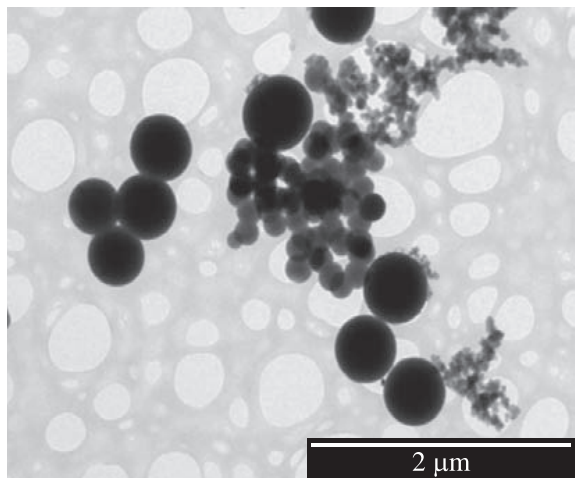

(f)

Figure 1. TEM images of the samples: a) 0; b) 5; c) 15; d) 30; and e) 45 minutes after initiation TEOS hydrolysis and condensation; and f) the sample prepared without MPTMS. 
The TEM image of the sample prepared with the simultaneous addition of the precursors ( 0 minute) revealed the formation of nanoparticles. The TEM images of the samples prepared after waiting 5, 15, 30, and 45 minutes before MPTMS addition and of the sample without MPTMS indicated the formation of agglomerated, overlapping, and dense spherical nanoparticles of different sizes. A comparison of the sample without added MPTMS and the one prepared with the addition of MPTMS after 45 minutes revealed a similar morphology but different particle sizes. The particles around silica nanoparticles may indicate the continuous formation of primary nuclei during the reaction ${ }^{14}$.

An analysis by electron diffraction confirmed the amorphousness of all the samples. The absence of MPTMS was found to prolong the hydrolysis time of TEOS and to lead to the formation of smaller particles. Particle size was probably influenced not only by the absence of MPTMS but also by the subsequent addition of $\mathrm{Eu}^{3+}$ ions to the TEOS.

The samples to which MPTMS was added after 5 and 30 minutes of TEOS condensation were examined by energy dispersive X-ray spectroscopy (EDS), to confirm the presence of $\mathrm{Si}$ and $\mathrm{O}$ atoms in the particles. The materials surrounding these silica nanopaticles (Figure 1) can be ascribed to organic groups derived from MPTMS. The EDS spectra confirm the presence of carbon atoms in these materials, as well as $\mathrm{Si}, \mathrm{O}$, and $\mathrm{Eu}$.

Table 1 depicts the range of silica nanoparticle sizes obtained by the sol-gel process.

The nanoparticle sizes were found to increase in the samples containing organosilane added after $0,5,15,30$, and 45 minutes. There was a tendency toward the formation of larger and less homogeneous particles of different sizes as the TEOS hydrolysis and condensation time increased.

The sample containing MPTMS added after 45 minutes showed the growth and formation of particles (Figure 1e) with sizes of about 370,510 , and $620 \mathrm{~nm}$. The sample which did not contain added MPTMS also evidenced the formation of particles of around 200, 470 and $530 \mathrm{~nm}$, although they were smaller than those of the samples containing MPTMS added after 45 minutes The other samples containing MPTMS added after 5, 15, and 30 minutes also revealed the growth of particles of various sizes, but there was no formation of isolated particles.

A study on particle growth ${ }^{1}$ stated that if the amount of TEOS exceeds a critical value, a second population of particles may appear. Their experimental and TEM observations indicated that the addition of MPTMS can stop the hydrolysis and condensation reaction of alkoxide TEOS instead of leading to particle growth. The TEM image of our 0 minute sample appears to show TEOS hydrolysis and condensation before the initial supersaturation of silica. After the initial supersaturation reached a critical level, primary silica nuclei were formed and aggregated rapidly, forming stable particles, as indicated in our TEM image of the 5 minutes sample. These stable particles grew as the TEOS hydrolysis time increased (15 and 30 minutes). According to Bogush and Zukoski, nucleation occurs continuously during the reaction ${ }^{13}$. In this case, if the rate of consumption of supersaturation in the growth of primary particles (nuclei) is sufficient to exhaust the rate of supersaturation generated by the reaction, then formation of secondary particles ensues. Thus, primary particles grow proportionately, resulting in larger and monodispersed particles. However, when the rate of generation of supersaturation is greater than the rate of consumption for growth, supersaturation of the solution increases and exceeds the critical level, resulting in the formation of secondary particles and leading to a multidispersity of particle sizes $^{13}$, as indicated in our TEM image of the 45 minutes sample. The imbalance between the rates of supersaturation and consumption for the growth of primary particles can probably be attributed to greater stability of the particles due to repulsion effects, after a critical size is reached ${ }^{14}$. Thus, the rate of consumption for the growth of these particles may decrease, while the rate of generation of supersaturation remains constant, causing the nuclei generated by supersaturation to aggregate and stable secondary particles to form.

Figure 2 presents the thermogravimetric curves (TG) of all the samples.

The TG curves of all the samples presented two stages of decay. The first stage, up to $200{ }^{\circ} \mathrm{C}$, can be ascribed to solvent or water molecules adsorbed on the surface of the silica. The mass loss in this region was accompanied by an endothermic transformation.

The second stage of decay occurred between 300 and $650{ }^{\circ} \mathrm{C}$ and can be attributed to the organic matter in the methacrylate group of the MPTMS, the incomplete hydrolysis of alkoxide TEOS, or the residual $-\mathrm{OH}$ groups on the surface of the silica.

Table 2 lists the percentage of mass loss as a function of temperature, for all the samples.

The loss of organic matter decreased as the time elapsed before MPTMS addition increased, probably because a larger amount of organic material was eliminated during the washing process. The longer waiting time before the addition of MPTMS may favor the separation of the component. Furthermore, it is probable that the longer the delay before the addition of organosilane MPTMS, the greater the increase in the TEOS alkoxide hydrolysis and condensation time, thereby resulting in larger particle sizes and reducing the amount of organic residue.

Table 1. Silica nanoparticle sizes as a function of TEOS hydrolysis time.

\begin{tabular}{cc}
\hline Samples (minutes) & Approximate sizes $(\mathrm{nm})$ \\
\hline 0 & - \\
5 & $350-410$ \\
15 & $350-510$ \\
30 & $350-635$ \\
45 & $350-690$ \\
Without MPTMS & $200-530$ \\
\hline
\end{tabular}

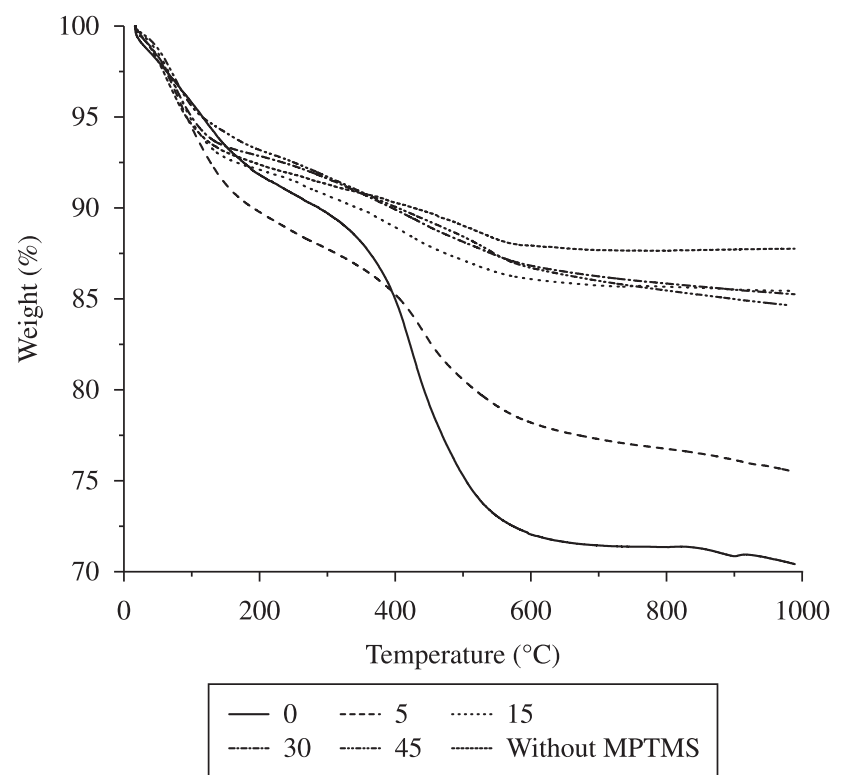

Figure 2. TG curves of the samples with MPTMS added after 0, 5, 15, 30, and 45 minutes after initiation of TEOS hydrolysis and condensation, and without MPTMS. 
Table 2. Percentage of mass loss as a function of temperature and MPTMS addition time.

\begin{tabular}{ccc}
\hline Samples (minutes) & Up to $200{ }^{\circ} \mathrm{C}(\%)$ & 300 to $650{ }^{\circ} \mathrm{C}(\%)$ \\
\hline 0 & 7.58 & 20.46 \\
5 & 10.12 & 10.08 \\
15 & 6.94 & 6.01 \\
30 & 6.70 & 5.18 \\
45 & 6.69 & 5.49 \\
Without MPTMS & 7.27 & 3.48 \\
\hline
\end{tabular}

A comparison of the 0 and 5 minutes samples revealed an increase in the loss of adsorbed solvent and water molecules and a reduction in the loss of organic molecules. The formation of primary nuclei altered the silica morphology. Condensation and the resulting release of water molecules and solvent were probably greater. With the formation of primary nuclei, further condensation probably occurs, followed by the release of water molecules and solvent. The 15, 30, and 45 minutes samples exhibited a decrease in stable mass loss in the two regions. As the infrared absorption spectra indicate, the organic matter originating from MPTMS decreased with increasing delay before addition of the organosilane, and it disappeared altogether in the 30 and 45 minutes samples. It is probable that the organic matter remaining in these samples was due to incomplete TEOS hydrolysis and condensation.

On the basis of the synthesis of spherical silica particles modified with methacrylate groups, Nassor et al. ${ }^{7}$ reported that the increase in the sample heat treatment temperature increased the degree of condensation of the alkoxide groups. According to Brinker and Scherer ${ }^{14}$, in the absence of mass loss, the exothermic densification of the silica skeleton is evidence of structural relaxation. Mass loss and shrinkage are competitors and can be attributed primarily to the elimination of organic matter (mostly lost mass), polymerization (contraction and weight loss), and structural relaxation (contraction only). The contraction can be attributed to the condensation reactions that occur on the surface of the inorganic network. Consequently, the network becomes denser by structural relaxation, an irreversible process in which the free energy decreases due to bond restructuring and rearrangements not associated with weight loss ${ }^{14}$.

The $\mathrm{Eu}^{3+}$ excitation and emission spectra recorded for all the samples are displayed in Figures 3 and 4, respectively.

The band ascribed to the ${ }^{7} \mathrm{~F}_{0}$ (fundamental level) to ${ }^{5} \mathrm{~L}_{6}$ (excitation level) transition was observed in the $0,5,15$, and 30 minutes, samples, but it was absent from the spectra of the sample containing MPTMS added after 45 minutes and the sample without MPTMS. In $\mathrm{SiO}_{2}: \mathrm{Eu}^{3+}$, Caiut et al. ${ }^{16}$ observed the O-Eu charge-transfer state (CTS), which was not detected in our system.

Figure 4 shows the emission spectra of $\mathrm{Eu}^{3+}$ ions in the matrix, where the excitation wavelength was recorded at $392 \mathrm{~nm},{ }^{5} \mathrm{~L}_{6}$ level.

The luminescence spectra for $\mathrm{Eu}^{3+}$ ions in the hybrid materials display a broad emision with maximum at around $515 \mathrm{~nm}$, whose intensity decreases as the pre-hydrolysis time increases. This band is observed for siloxanes and is assigned to electron-hole recombination due to the defects at the surface of the siloxane particles ${ }^{17}$. The $\mathrm{Eu}^{3+}$ emission bands in these spectra were characterized by the nonhomogenous distribution of the ion in the silica matrix ${ }^{18-20}$. The characteristic emission bands of $\mathrm{Eu}^{3+}$ ions were observed in the region of $580,590,612,650$, and $700 \mathrm{~nm}$, corresponding to the electronic transitions from the excited ${ }^{5} \mathrm{D}_{0}$ to the fundamental ${ }^{7} \mathrm{~F}_{\mathrm{J}(\mathrm{J}=0,1,2,3 \text { and } 4)}$ levels. The band corresponding to ${ }^{5} \mathrm{D}_{0} \rightarrow{ }^{7} \mathrm{~F}_{0}$ transitions at $579 \mathrm{~nm}$ is due to sites without inversion centers occupied by $\mathrm{Eu}^{3+}$ ions $^{21}$.

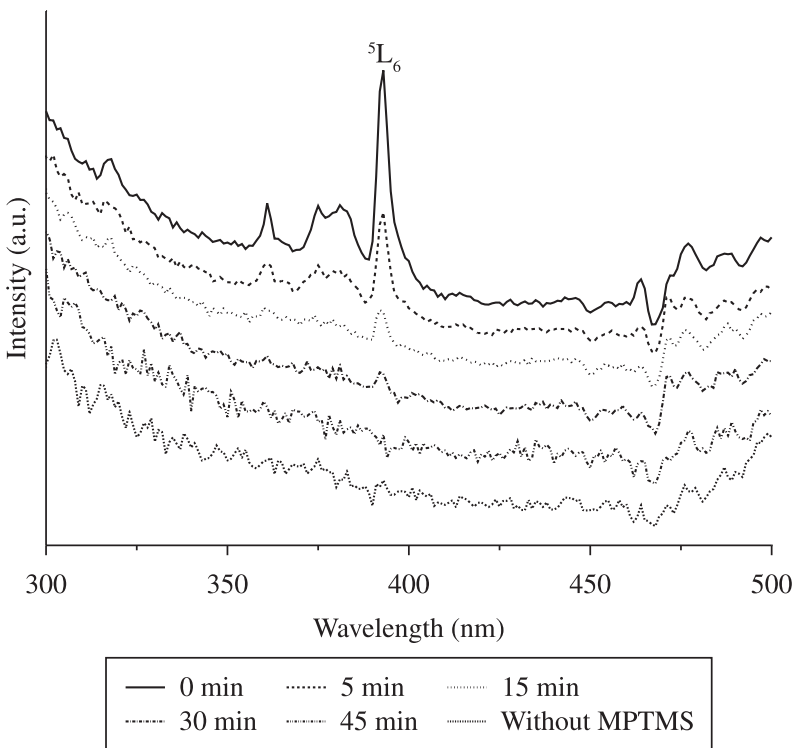

Figure 3. Excitation spectra of the $\mathrm{Eu}^{3+}$ ions in the silica matrix of the samples containing MPTMS added after $0,5,15,30$, and 45 minutes after initiation of TEOS hydrolysis and condensation, and the sample without MPTMS, $\lambda_{\mathrm{em}}=612 \mathrm{~nm}$.

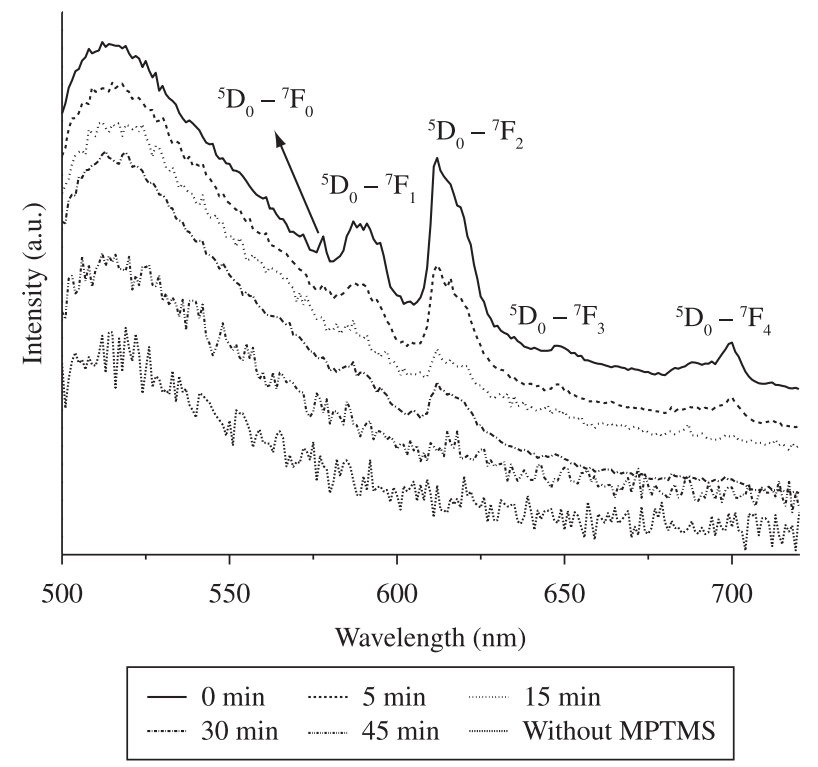

Figure 4. Emission spectra of the $\mathrm{Eu}^{3+}$ ions in the silica matrix of the samples containing MPTMS added after $0,5,15,30$, and 45 minutes after initiation of TEOS hydrolysis and condensation, and the sample without MPTMS, $\lambda_{\text {exc }}=392 \mathrm{~nm}$.

The samples containing MPTMS added after 0, 5, and 15 minutes presented bands corresponding to the transition from the excited ${ }^{5} \mathrm{D}_{0}$ to the fundamental ${ }^{7} \mathrm{~F}_{\mathrm{J}(\mathrm{J}=0,1,2,3 \text { and } 4)}$ levels. These samples displayed a band in the region of $579 \mathrm{~nm}$, suggesting the presence of a possible site without an inversion center, which can be confirmed by the presence of a ${ }^{5} \mathrm{D}_{0} \rightarrow{ }^{7} \mathrm{~F}_{2}$ transition band that should be absent from sites with inversion centers, and can be attributed to the presence of more than one site of $\mathrm{Eu}^{3+}$ ion due to large emission bands.

$\mathrm{The}^{3+}$ ion emission intensity decreased with the timing of the addition of MPTMS because of the increased amount of water in the samples, as indicated by the thermogravimetric curve. It is possible 
that the $\mathrm{Eu}^{3+}$ ions surrounding the silica particles reduce their emission intensity because of vibrational losses. However, it is more likely that most of these ions were eliminated during the washing process, since they had been added to the sample together with the MPTMS. The sample without MPTMS did not show emission from the ion, probably due to energy loss by vibrational modes of $-\mathrm{OH}$ groups on the surface of the silica, resulting from the incomplete condensation of the TEOS alkoxide. The presence of - $\mathrm{OH}$ groups interferes directly in the emission of $\mathrm{Eu}^{3+}$ ion. Because of the electric-dipole character of the $\mathrm{Eu}^{3+}$, the intensities of the ${ }^{5} \mathrm{D}_{0} \rightarrow{ }^{7} \mathrm{~F}_{0}$ and ${ }^{5} \mathrm{D}_{0} \rightarrow{ }^{7} \mathrm{~F}_{2}$ transitions are strongly dependent on the surrounding ${ }^{22}$. The band corresponding to the ${ }^{5} \mathrm{D}_{0} \rightarrow{ }^{7} \mathrm{~F}_{1}$ transition presents a magnetic dipole nature and its intensity is not affected by the surroundings. Therefore, it can be considered a standard to measure the relative intensity of the other bands ${ }^{22}$. The fact that the intensity ratio presents lower values for the hybrids indicates that $\mathrm{Eu}^{3+}$ ions are located in sites with a lower symmetry, and these results are observed in other works ${ }^{23-25}$.

The infrared spectra of the samples are shown in Figure 5

The spectra of the materials display bands associated with the vibrations of both inorganic and organic structural units. A slight difference in the spectra can be observed in relation to the number and relative intensity of the bands.

In Figure 5, the spectra of the samples containing MPTMS added after $0,5,15,30$, and 45 minutes, and without MPTMS presented bands ascribed to vibrational modes characteristic of silica. The most intense band, at about $1100 \mathrm{~cm}^{-1}$, is associated with the asymmetric stretching vibrations of the Si-O-Si bridges. Vibrations of the siliconoxygen bridges are also responsible for the bands at about $800 \mathrm{~cm}^{-1}$ (symmetric stretching vibrations) and at about $463 \mathrm{~cm}^{-1}$ (bending vibrations $)^{26}$. The bands related to the $\mathrm{Si}-\mathrm{O}-\mathrm{Si}$ bridges were found to increase in intensity according to the timing of MPTMS addition, probably due to increased TEOS hydrolysis and condensation. According to Chen and Brauer ${ }^{27}$, the band at $1868 \mathrm{~cm}^{-1}$ can be attributed to $\mathrm{Si}-\mathrm{O}-\mathrm{Si}$ bridges in the silica network.

The band at $1632 \mathrm{~cm}^{-1}$ can be ascribed to water bending. Vibrations in the region of $3000-3750 \mathrm{~cm}^{-1}$ were observed and attributed to $-\mathrm{OH}$ stretching on the silica surface and also to water left over from the synthesis, indicating incomplete TEOS condensation.

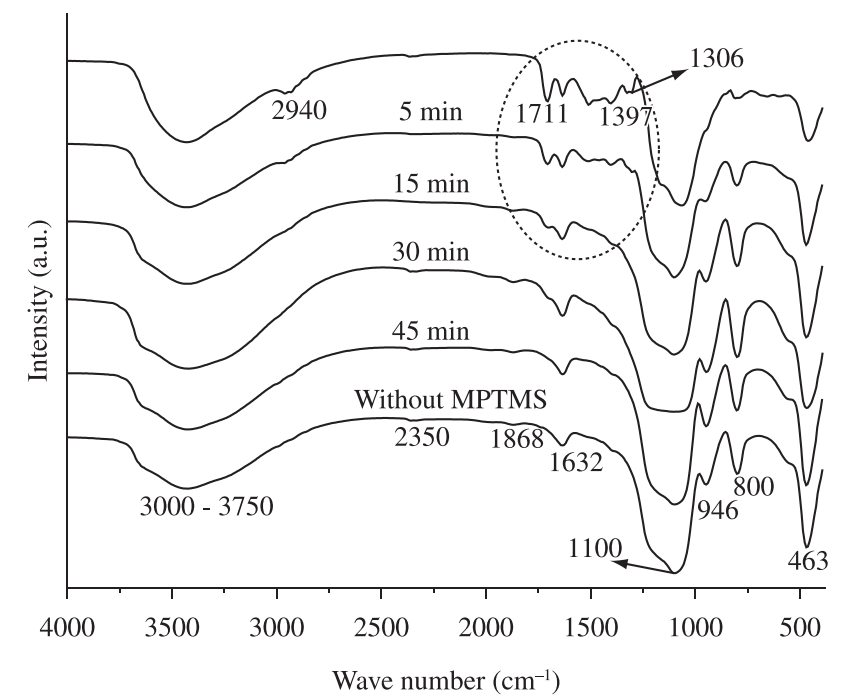

Figure 5. Infrared spectra of the samples containing MPTMS added after 0, 5, 15,30 , and 45 minutes after initiation of TEOS hydrolysis and condensation, and without MPTMS.
The band related to $\mathrm{Si}-\mathrm{OH}$ group vibrations was observed at about $946 \mathrm{~cm}^{-1[26,28]}$. With regard to the absorption spectra, note that the intensity of this band increases with longer time elapsed before MPTMS addition, indicating greater hydrolysis and incomplete condensation of TEOS.

According to Vinod et al., the band at $2350 \mathrm{~cm}^{-1}$ in organosilane polymers represents defects on the silica surface ${ }^{29}$. This band showed low intensity. The bands corresponding to the organic matter, $\mathrm{C}=\mathrm{O}$ at $1711 \mathrm{~cm}^{-1},{\mathrm{C}-\mathrm{COO}^{-}}^{-}$at $1306 \mathrm{~cm}^{-1}, \mathrm{CH}_{2}=$ at $1397 \mathrm{~cm}^{-1}$, and $\mathrm{CH}-$ at $2940 \mathrm{~cm}^{-1[30,31]}$, decreased in intensity as the timing elapsed before MPTMS addition increased, confirming the thermal analysis results. All the samples contained residual organic matter originating from either MPTMS or incomplete TEOS hydrolysis. However, the amount of MPTMS-related organic matter decreased in the 5 and 15 minutes samples and was absent from the 30 and 45 minutes samples. This indicates that a longer wait prior to the addition of MPTMS may allow for a greater separation of the component, and for a larger amount of MPTMS-related organic matter to be eliminated during washing of the material.

\section{Conclusion}

The methodology applied here allowed for the formation of spherical silica nanoparticles of different sizes. The particle size and morphology were found to be directly dependent on the TEOS hydrolysis and condensation time. The intensity of luminescence decreased as the timing before addition of $\mathrm{Eu}^{3+}$ ions and MPTMS increased. The longer wait before these additions probably enabled the MPTMS-related organic material and the $\mathrm{Eu}^{3+}$ ions to be removed during washing; this was confirmed by infrared spectroscopy and thermal analysis.

The samples showed particle morphology and size close to the expected values, but the methacrylate groups were probably not bound to the silica particles. The absence of organosilane MPTMS gave rise to the longest TEOS hydrolysis and condensation time and the formation of smaller particles.

It can be concluded that the parameters of the sol-gel process, such as hydrolysis and condensation time, and the order in which the reagents are added can affect the particle size, size distribution and morphology, as well as the luminescence of $\mathrm{Eu}^{3+}$ ions.

\section{Acknoledgements}

The authors acknowledge FAPESP, CNPq, and CAPES (Brazilian research funding agencies) for the financial support of this work.

\section{References}

1. Rao KS, El-Hami K, Kodaki T, Matsushige K and Makino K. A novel method for synthesis of silica nanoparticles. Journal of Colloid and Interfase Science. 2005; 289:125-131.

2. Caetano BL, Rocha LA, Molina EF, Rocha ZN, Ricci GP, Calefi PS et al. Cobalt aluminu silicate complexes prepared by the non-hydrolytic sol-gel route and their catalytic activity in hydrocarbon oxidation. Applied Catalysis A. 2006; 311:122-134.

3. Rocha LA, Molina EF, Ciuffi KJ, Calefi PS and Nassar EJ. Eu (III) as a probe in titânia thin films: the effect of temperature. Materials Chemistry and Physics. 2007; 101(1):238-241.

4. Sanchez C, Julián B, Belleville P and Popall M. Applications of hybrid organic-inorganic nanocomposites. Journal of Materials Chemistry, 2005; 15:3559-3592.

5. Wilson LS, Zhang K and Antonucci JM. Systematic variation of interfacial phase reactivity in dental nanocomposites. Biomaterials. 2006; 26:5095-5103. 
6. Stober W, Fink A and Bohn E. Controlled growth of monodisperse silica spheres in the micron size range. Journal of Colloid and Interfase Science, 1968; 26:62-69.

7. Nassor ECO, Nassar EJ, Avila LR, Pereira PFS, Cestari A, Luz LM et al. Spherical hybrid silica particles modified by methacrylate groups. Journal of Sol-Gel Science and Technology. 2007; 43(1):21-26.

8. Branda F, Silvestri B, Luciani G and Costantini A. The effect of mixing alkoxides on the Stober particles size. Colloids and Surfaces A: Physicochemistry Engeneering Aspects. 2007; 299:252-255.

9. Bermudez VZ, Carlos LD, Duarte MC, Silva MM, Silva CJR, Smith $\mathrm{MJ}$ et al. A novel class of luminescent polymers obtained by the sol-gel approach. Journal of Alloys and Compounds, 1998; 275-277:21-26.

10. Nassar EJ, Avila LR, Pereira PFS, Nassor ECO, Cestari A, Ciuffi KJ et al. Fenilssilicato dopado com Eu III obtido pelo método sol-gel. Química Nova. 2007; 30(7):1567-1572.

11. Costa CAR, Leite CAP and Galembeck F. Size dependence of Stober silica nanoparticle microchemistry. Journal Physical and Chemistry B. 2003; 107:4747-4755.

12. Green DL, Lin JS, Lam Y, HuM Z-C, Schaefer DW and Harris MT. Size, volume fraction, and nucleation of Stober silica nanoparticles. Journal of Colloid and Interfase Science. 2003; 266:346-358.

13. Bogush GH and Zukoshi CF. Sudies of the kinetics of the precipitation of uniform silica particles through the hydrolysis and condensation of silicon alkoxides. Journal of Colloid and Interfase Science. 1991; 142(1):1-18.

14. Brinker CJ and Scherer GW. Sol-Gel Science, The Physcs and Chemistry of Sol-Gel Processing. San Diego: Academic Press; 1990.

15. Chen S, Dong P, Yang G and Yang J. Kinetics of formation of monodisperse colloidal particles through the hydrolysis and condensation of tetraethylorthosilicate. Industrial Enginnering Chemistry Research. 1996; 35:4487-4493.

16. Caiut JMA, Bazin L, Mauricot R, Dexpert H and Ribeiro SJL. DexpertGhys J, Luminescente nano-composites generated from a spray. Journal of Non-Crystalline Solids. 2008; 354:4860-4864.

17. Carlos LD, Ferreira RAS, Bermudez VZ and Ribeiro SJL. Full-colorphosphores from amine functionalized crosslinked hybrids lacking metal activator ions. Advance Functional Materials. 2001; 11(2):111-115.

18. Hazenkamp MF, Van der Veen AMH, Feiken W and Blasse G. Hydrated rare-earth-metal ion-exchanged zeolite a: characterization by luminescence spectroscopy. Part 2.-The Eu ${ }^{3+}$ ion. Journal Chemistry Society Faraday Translation. 1991; 88(1):141-144.

19. Rice DK and Deshaser LG. Spectral broadening of europium ions in glass. Physics Review B. 1969; 186:387-392.

20. Reisfeld R. Fluorescence and nonradiative relaxations of rare earths in amorphous media and on high surface area supports: a review. Journal of Eletrochemistry Society. 1984; 131:1360-1364.

21. Serra OA, Nassar EJ, Zapparolli G and Rosa ILV. Organic Complexes of Eu III Supported in Functionalyzed Silica Gel: Highly Luminescent Materials. Journal of Alloy and Compounds. 1994; 207-208:454-456.

22. Richardson FS. Terbium(III) and europium(III) ions as luminescent probes and stains for biomolecular systems. Chemical Review. 1982; 82:541-552.

23. Reisfeld R. Excited states and energy transfer from donor cations to rare earths in the condensed phase. Structuring Bonding, 1979; 30:65-97.

24. Caiut JMA, Rocha LA, Sigoli FA, Messaddeq Y, Dexpert-Ghys J and Ribeiro SJL. Aluminoxane-epoxi-siloxane hybrids waveguides. Journal of Non-Crystalline Solids. 2008; 354:4795-4799.

25. Avila LR, Nassor ECO, Pereira PFS, Cestari A, Ciuffi KJ, Calefi PS et al. Preparation and properties of europium-doped phosphosilicate glasses obtained by the sol-gel method. Journal of Non-Crystalline Solids. 2008; 354:4806-4810.

26. Wojtach K, Cholewa-Kowalska K, Laczka M and Olejinizak Z. Coloured organic-inorganic coating on glass. Optical Materials. 2005; 27:1495-1500.

27. Chen TM and Brauer GM. Solvent effects on bonding organo-silane to silica surfaces. Journal of Dental Research. 1982; 61(12):1439-1443.

28. Godoi RHM, Fernandes L, Jafelicci Jr M, Marques RC, Varanda LC and Davolos MR. Investigation of the systems silica and silica containing chromium in alcohol medium. Journal of Non-Crystalline Solids, 1999; 247:141-145.

29. Vinod PM, Bahnemann D, Rajamonhanan RB and Vijayamohanan K. A novel luminescent functionalized siloxane polymer. Journal Physics and Chemistry B. 2003; 107:11583-11588.

30. Anagnostopoulos T, Eliades G and Palaghias G. Composition, reactivity and surface interactions of three dental silane primers. Dental Materials. 1993; 9:182-190.

31. Skoog DA. Princípios de análise instrumental. 5. ed. Porto Alegre: Bookman; 2002. 